\title{
Optimal Filter Overdesign
}

\author{
Antônio C. M. de Queiroz and Luiz P. Calôba
}

\author{
Programa de Engenharia Elétrica - COPPE/DEEL \\ Universidade Federal do Rio de Janeiro \\ CP 68504, 21945-970 Rio de Janeiro, RJ, Brazil
}

\begin{abstract}
This paper presents a method for the overdesign of gain-shaping filters in an optimal way. The passband minima and the stopband maxima of a filter approximation are adjusted using an iterative method, with the objective of obtaining a special approximation, that satisfies exactly the specifications when the worst-case error in the filter transfer function due to errors on its components is considered.
\end{abstract}

\section{INTRODUCTION}

The classical filter approximations for filters with steep transition bands, the Chebyshev approximation for all-pole filters and the elliptic approximations for filters with finite transmission zeros, result in equal-ripple passbands, and in the case of the elliptic approximations, in also equal-ripple stopbands. These characteristics are necessary if the approximations are to provide the shortest possible transition bands for a given filter order and attenuation specifications.

It is recognized, however, that these optimal approximations are in many cases of impractical realization, because the precision needed in the component values for the realization of the filter by the desired structure is unattainable. This happens when errors in the filter component values can modify the filter transfer function in a way that violates the filter specifications.

The usual approach when this occurs is to overdesign the filter, by reducing the passband ripple, increasing the minimum stopband attenuation, and if necessary increasing the filter order. In some cases, this turns the approximation into something closer to the Butterworth approximation, that is the limit of the Chebyshev or elliptic approximations when the passband ripple is reduced and the stopband minimum attenuation increased. In a filter built with an LC doubly terminated structure, or any active simulation of one that preserves the sensitivity characteristics, this approach generally has success, because the passband and stopband sensitivities decrease in the process. Usually the overdesign is done simply by using more strict filter specifications, and obtaining an equal-ripple approximation that satisfies them.

An observation of the sensitivity characteristics of most filter structures realizing an equal-ripple approximation shows that the errors due to component value errors are not uniform in frequency, but are higher at the passband and stopband borders. A reason for this is that in these regions the transfer function is determined by groups of poles or zeros closely spaced, and errors in their relative positions cause large errors in the transfer function magnitude.

Most high-precision filters are based on LC doubly terminated structures, or realized as active simulations of passive prototype filters with those structures, due to the very low sensitiv- ity to variations in element values attainable [1]. In an LC doubly terminated filter, the gain cannot exceed the one determined by maximum power transfer. In consequence, in a filter designed with maximum power transfer at the passband maxima, the passband errors due to small changes in component values can affect only the passband minima. This results in the sensitivities to all the LC element values being null at the passband maxima, and with peaks located somewhere between adjacent maxima. For equal-ripple approximations, these sensitivity peaks are higher close to the passband border. For the terminations, the sensitivities have a frequency-dependent part that follows the same pattern, and a frequency-independent part $( \pm 0.5)$ that affects only the "flat-loss" of the filter, that in most cases can be ignored. At the stopband, the error analysis by sensitivity presents the artifact of infinite sensitivities to the values of the elements forming the zeros at the transmission zeros frequencies [2]. The errors that are really important are the ones at the peaks of the stopband "humps". For equal-ripple approximations, these errors are higher close to the stopband border. Figures 1-2 illustrate these characteristics, by depicting the gain characteristic of a 7th-order elliptic low-pass filter ( $1 \mathrm{~dB}$ passband ripple, $50 \mathrm{~dB}$ minimum stopband attenuation, and passband border at $1 \mathrm{rad} / \mathrm{s}$ ) realized by a standard LC doubly terminated ladder structure, with the gain statistical deviation when uncorrelated $5 \%$ errors in all the element values are considered. The error was computed by eq. (1), and is shown subtracted in the passband and added in the stopband, with the terminations' sensitivities discounted of the frequency-independent part.

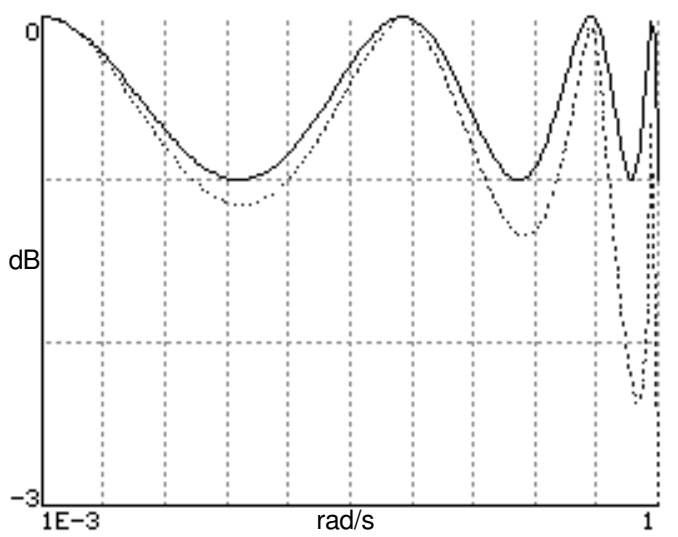

Fig. 1. Passband gain curve of a normalized elliptic 7th-order low-pass filter, and the gain curve with the statistical deviation due to $5 \%$ errors in all the element values subtracted, with the flat-loss error discounted. The maximum gain was normalized to $0 \mathrm{~dB}$, as in the other figures. 


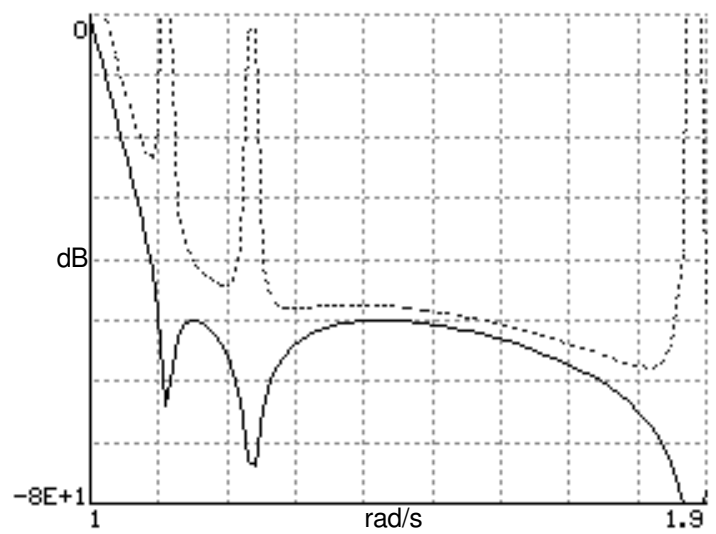

Fig. 2. Stopband (beginning) gain curve for the same filter, shown also with the statistical deviation added, discounted of the flat-loss error.

If the passband or stopband ripple is irregular, it can be shown that there is a direct proportion between the depth of the passband "valleys" and stopband "humps" and the error at their frequencies. This relation is used in the optimal overdesign method described below.

\section{OPTIMUM OVERDESIGN}

The gain specifications that are certainly satisfied by a filter can be obtained by adding or subtracting the error curves from the nominal gain curve, as done in figs. 1-2. The errors can be computed by several methods. The one used in the examples in this paper is the gain statistical deviation, computed by:

$$
\Delta|T(j \omega)|=\frac{20}{\operatorname{Ln}(10)} \sqrt{\sum_{i}\left(V_{i} \operatorname{Re} S_{x_{i}}^{T(j \omega)}\right)^{2}} \mathrm{~dB}
$$

where $T(j \omega)$ is the transfer function, $x_{i}$ are the filter component values, and $V_{i}$ their relative tolerances, $\Delta x_{i} / x_{i}$.

In an LC doubly terminated filter, it is easy to eliminate from the errors the flat-loss errors, that affect only the gain of the filter and are easy to compensate. They are caused only by errors in the terminations at the frequencies where maximum power transfer occurs. With the sensitivities of the terminations discounted of this value, the measure (1) reduces to zero at the maximum power transfer frequencies, that is, at the passband gain maxima for a well-designed filter. With this discounted error used, the passband gain limits are obtained by subtracting the error curve from the nominal gain curve, since the maximum power transfer prevents any increase in the passband gain.

In the stopband, the maximum gain (minimum attenuation) limit is obtained by adding the error curve to the nominal gain curve. The discount of the flat-loss error can also be used. The infinite sensitivities at the transmission zeros can be ignored, by considering only the error at the stopband maxima and borders, or by using "slope-normalized" sensitivities [2].

When the errors in an overdesigned equal-ripple filter are considered, clearly the worst-case (in this case in statistical sense) resulting filter is not equal-ripple at all. It is possible, however, to use the fact that errors are higher at the passbands and stopbands limits to obtain a more selective overdesigned filter. This can be done by computing the overdesigned approximation with irregular passband and stopband ripples, in a way that makes the worst-case resulting filter equal-ripple. The idea is exemplified in the example below.

\section{EXAMPLE I}

A 7th-order elliptic filter realized as a doubly terminated LC ladder presents the passband and stopband gains and errors shown in figs. 1-2. The objective is to obtain a similar filter, that in the worst case is equal-ripple in the passband and stopband. An algorithm for obtaining an optimally overdesigned filter is:

1. Compute the worst-case gain curve, as the filter gain curve $(\mathrm{dB})$, minus the flat-loss $(-6.0206 \mathrm{~dB}$ in the example), minus the gain statistical deviation $(\mathrm{dB})$ in the passband, and plus the gain statistical deviation in the stopband, as discussed in the last section. If it is equal-ripple, stop. Else, call $E_{i}$ the gains at the passband minima of this composite curve:

$$
E_{i}=\left|T\left(j \omega_{i}\right)\right|-\text { flat }- \text { loss }+\Delta\left|T\left(j \omega_{i}\right)\right| \mathrm{dB}
$$

and call $E_{i}^{\prime}$ the gains of the composite curve at the stopband gain maxima:

$$
E_{i}^{\prime}=\left|T\left(j \omega_{i}\right)\right|-\text { flat - loss }+\Delta\left|T\left(j \omega_{i}\right)\right| \mathrm{dB}
$$

Call $A_{i}$ the designed gains $(\mathrm{dB})$ of the filter at the passband minima and $A_{i}{ }^{\prime}$ the designed gains at the stopband maxima, both without considering the flat-loss.

2. Compute new $A_{i}=\left(-A_{\max } \times A_{i}\right) / E_{i}$, and $A_{i}^{\prime}=\left(-A_{\min } \times A_{i}^{\prime} \not H_{i}^{\prime}\right.$, the gains at the passband minima and at the stopband maxima to be used as design parameters in the next iteration. $A_{\max }$ is the specified maximum passband attenuation and $A_{\min }$ is the specified minimum stopband attenuation $(\mathrm{dB})$. These expressions assume that the direct proportions among the $E_{i}$ and $E_{i}^{\prime}$, and the corresponding $A_{i}$ and $A_{i}^{\prime}$ are linear and independent, what is approximately true, specially at the passband.

3. Compute a new approximation, with irregular ripple, with passband minima $A_{i}$ and stopband maxima $A_{i}^{\prime}$ Suitable algorithms were described in [3], and specially in [4]. Compute the new values for the filter network.

4. Return to 1 .

TABLE I

Nominal passband minimum gains $A_{i}$, stopband maximum gains $A_{i}{ }^{\prime}$, and corresponding gains with worst-case error considered, $E_{i}$ and $E_{i}{ }^{\prime}$, for the six first steps of the optimization algorithm of the example.

\begin{tabular}{|l|lll|lll|}
\hline & $-A_{1} /-E_{1}$ & $-A_{2} /-E_{2}$ & $-A_{3} /-E_{3}$ & $-A_{1}^{\prime} /-E_{1}^{\prime}$ & $-A_{2}^{\prime} /-E_{2}^{\prime}$ & $-A_{3}^{\prime} /-E_{3}^{\prime}$ \\
\hline \multirow{2}{*}{1} & 1 & 1 & 1 & 50 & 50 & 50 \\
& 1.154 & 1.341 & 2.382 & 40.63 & 47.51 & 49.08 \\
\hline \multirow{2}{*}{2} & 0.866 & 0.746 & 0.420 & 61.78 & 52.62 & 50.93 \\
& 1.012 & 1.049 & 1.154 & 48.64 & 50.10 & 50.00 \\
\hline \multirow{2}{*}{3} & 0.856 & 0.711 & 0.364 & 63.51 & 52.51 & 50.93 \\
& 1.001 & 1.009 & 1.037 & 49.23 & 50.01 & 50.00 \\
\hline \multirow{2}{*}{4} & 0.855 & 0.705 & 0.351 & 64.50 & 52.50 & 50.93 \\
& 1.000 & 1.002 & 1.008 & 49.52 & 50.00 & 50.00 \\
\hline \multirow{2}{*}{5} & 0.855 & 0.703 & 0.348 & 65.13 & 52.49 & 50.93 \\
& 1.000 & 1.000 & 1.001 & 49.68 & 50.00 & 50.00 \\
\hline \multirow{2}{*}{6} & 0.855 & 0.703 & 0.348 & 65.55 & 52.49 & 50.93 \\
& 1.000 & 1.000 & 1.000 & 49.77 & 50.00 & 50.00 \\
\hline
\end{tabular}

Table I shows the obtained results for 6 iterations, starting from a normalized filter with $1 \mathrm{~dB}$ passband ripple and $50 \mathrm{~dB}$ minimum stopband attenuation. $5 \%$ tolerances in the filter components were assumed. It can be observed that the convergence is slower at the passband and stopband borders. Figures 3-6 show the 
comparison of the optimum overdesigned filter (figs. 3, 5) with a conventional equal-ripple overdesign, obtained by the same algorithm, but considering only $A_{3}, A_{1}{ }^{\prime}$ and $E_{3}, E_{1}{ }^{\prime}$ (this results in a 0.41 $\mathrm{dB}$ passband ripple and $57.43 \mathrm{~dB}$ minimum stopband attenuation.), and with a 8th-order modified overdesigned elliptic filter (with two transmission zeros at $\infty$, the next that admits a simple ladder realization), obtained in the same way, resulting in $0.33 \mathrm{~dB}$ passband ripple and $60.61 \mathrm{~dB}$ minimum stopband attenuation (figs. 4,6 ).

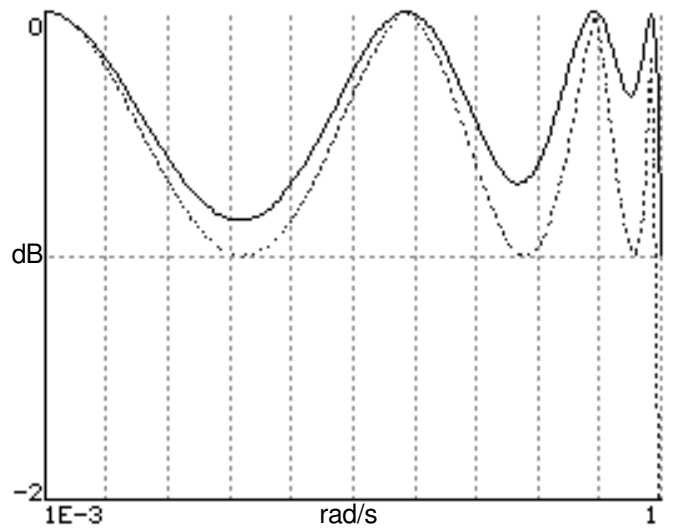

Fig. 3. Optimum overdesigned filter passband gain, with flat-loss discounted, shown also with the gain statistical deviation subtracted.

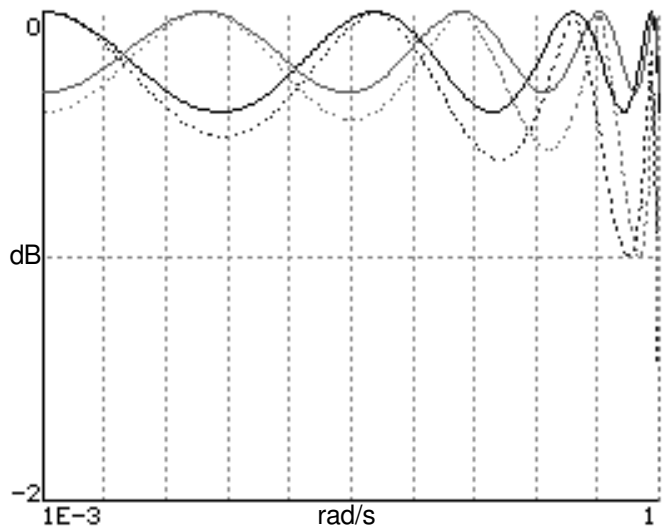

Fig. 4. Equal-ripple 7th-order and 8th-order overdesigned filters passband gains, as in fig. 3 .

The optimum overdesigned filter is more selective than the equal-ripple one of the same order, and slightly less selective that the 8th-order uniform-ripple overdesigned filter, as can be observed in figs. 5-6.

A frequency scaling shall be done to center the transition band of the overdesigned filter in the specifications (not done in the example). Note that, with this frequency scaling the method would find a solution for any values of $A_{\max }$ and $A_{\min }$. The limit solution would be a Butterworth approximation, frequency scaled as needed to reduce the worst-case passband and stopband errors. Of course, the order could have to be increased to satisfy the transition band width specification, and the order increase would increase the error at the passband and stopband borders. In critical cases a solution can be inexistent, by this or by any other overdesign method.

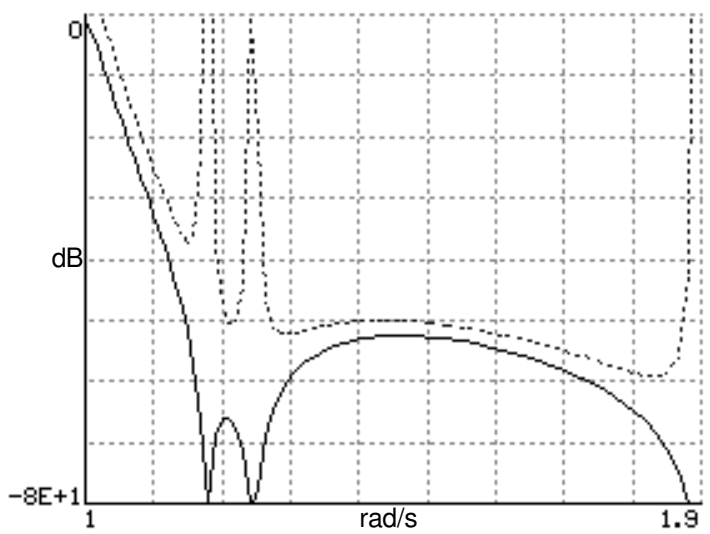

Fig. 5. Optimum overdesigned filter stopband gain, with flat-loss discounted, shown also with the gain statistical deviation added.

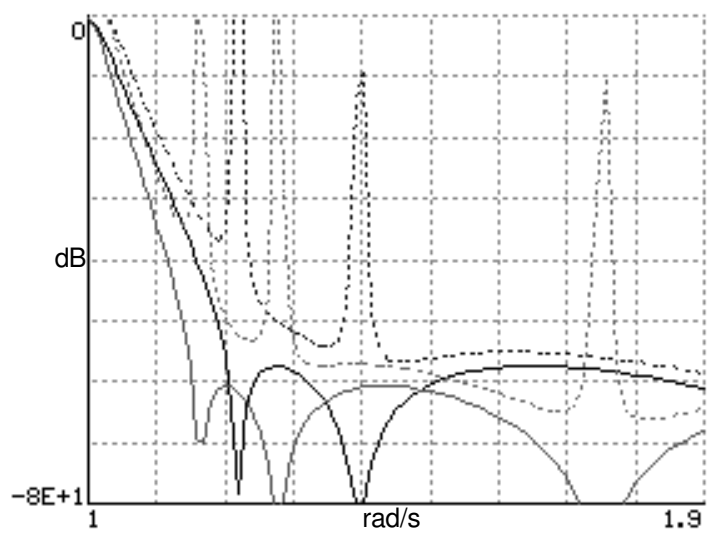

Fig. 6. Uniform-ripple 7th-order and 8th-order overdesigned filters stopband gains, as in fig. 5.

\section{DIRECT OPTIMIZATION}

For the implementation of the proposed method, a computer program was used to compute and locate the passband and stopband gain limits, with errors computed by sensitivity analysis, done by the "adjoint network" method. The filter synthesis steps were done by another program, that implements the algorithm described in [4]. Although rather complicated, this procedure is able to design the filters with great precision, in a reasonable time, and without numerical problems. A simpler approach was investigated, using direct optimization or the filter structure, using a simple gradient optimizer included directly in the analysis program. The algorithm is:

1. Analyze the filter, as in the first step of the previous algorithm (locating passband minima and stopband maxima, with error considered), but locate also the passband maxima of the gain curve alone.

2. For each of the frequencies of these extremes, and also for the passband edge frequency, write an equation:

$$
\sum_{i=1}^{n} \operatorname{Re}\left(S_{x_{i}}^{T(j \omega)}\right) \frac{\Delta x_{i}}{x_{i}}=\frac{\operatorname{Ln}(10)}{20}\left(|T(j \omega)|_{\text {desired }}-|T(j \omega)|_{\text {with error }}\right)
$$

where the $x_{i}$ are a set of $n$ elements of the filter, usually all the reactive elements. The term at the right side is the error in $\mathrm{Ne}$ pers between the desired gain and the obtained gain (with er- 
ror considered) with the present filter structure. This results in a system of linear equations for $\Delta x_{i} / x_{i}$, that when solved gives corrections for the element values that transform the present filter into another with a gain curve closer to the desired one. The assumption that the error extremes are proportional to the gain extremes allows the use of gain sensitivities to compute the corrections.

3. For usual filter structures, the number of extremes needed for the complete characterization of the filter gain curve, plus values that define frequency scaling and bandwidth, is precisely equal to the number of reactive elements. In these cases the system of equations (3) is directly solvable (if not singular). If there are more reactive elements than needed, or if the terminations are included among the $x_{i}$, the system (3), in the form $\mathbf{S v}=\mathbf{e}$, has more unknowns than equations, and there are many solutions. A convenient solution is obtained by transforming the system of equations into $\mathbf{S S}^{\mathrm{T}} \mathbf{z}=\mathbf{e}$, with $\mathbf{v}$ (the $\Delta x_{i} / x_{i}$ vector) recovered as $\mathbf{v}=\mathbf{S}^{\mathrm{T}} \mathbf{z}$. It can be shown that this transformation computes the vector $\mathbf{v}$ with the minimum possible modulus, that satisfies $\mathbf{S v}=\mathbf{e}$.

4. Update the element values by $x_{i} \leftarrow x_{i}\left(1+\Delta x_{i} / x_{i}\right)$, and return to 1.

This algorithm is simpler, and less computationally intensive than the previous one, since it avoids the synthesis of a new approximation, that is also done by optimization, and the filter synthesis. It presents, however, some numerical problems. The most serious is that the LC doubly terminated realization with maximum power transfer is an "extreme" solution for an approximation. The gain sensitivity matrix $\mathbf{S}$ becomes singular at the solution. It is obvious that the lines of the matrix corresponding to passband maxima are null, but there are also other relations that turn the matrix singular even if the maxima are substituted by other frequencies. The problem can be avoided by ignoring the equations of the maximum power transfer extremes when the gain is close enough to the desired value, with the transpose matrix technique (step 3 above) used to correct the number of unknowns in the system of equations. This problem does not exist for filters without maximum power transfer, but another problem appears: Because there is no relation among gain minima and error maxima, and the discounting of the flat-loss error can' be made, the number of extremes in the gain curve and in the gain-error curve in the passband can be different. For any filter, the overdesign of passband and stopband borders is also problematic, since the assumed proportionality between attenuation and error is not valid in these regions. The multiplication of the computed $\Delta x_{i} / x_{i}$ by a reduction factor (about 0.5 ) is usually necessary to force the convergence in these cases.

\section{EXAMPLE II}

This example illustrates the overdesign by direct optimization. A normalized 6th-order irregular band-pass filter, with passband between 1 and $2 \mathrm{rad} / \mathrm{s}$, with $1 \mathrm{~dB}$ ripple, and an inferior rejection band with 2 transmission zeros and minimum attenuation of 50 $\mathrm{dB}$ was computed directly by the optimization algorithm described, from an initial approximation with the correct shape, obtained by estimating empirically the element values.

The filter was overdesigned by the consideration of the errors at the two stopband maxima, the two passband minima, and the passband borders, with the terminations fixed, and 5\% tolerances assumed for all the elements. The final element values are listed in fig. 7. The obtained gain curve is shown in fig. 8 , with the passband detailed.

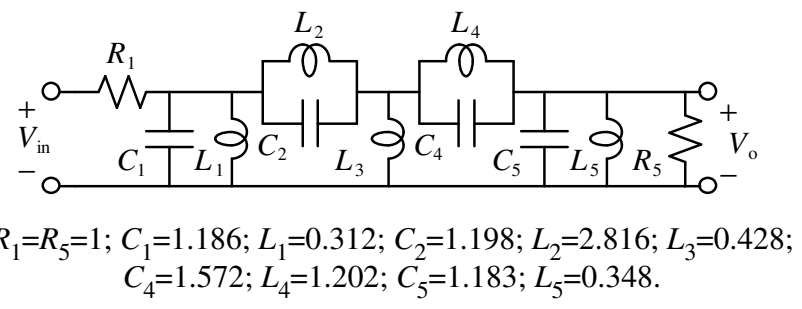

Fig. 7. Overdesigned irregular band-pass filter. Units in $\Omega, F$, and $H$.

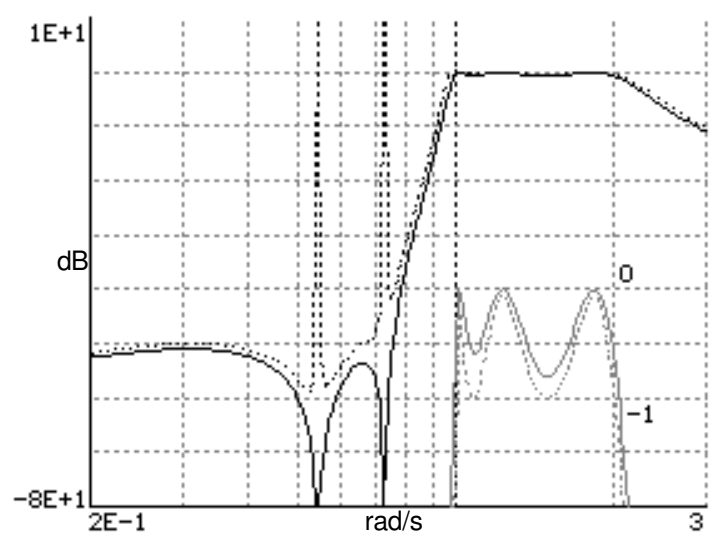

Fig. 8. Frequency response of the overdesigned irregular band-pass filter.

\section{CONCLUSIONS}

A method for optimized overdesign of gain-shaping filters with maximum-ripple passbands and stopbands was presented, with two algorithms described. The first algorithm is more precise, although more computationally intensive. The second algorithm is simpler and more general, but somewhat numerically problematic. The examples used the statistical deviation as a measure of the worst-case error. A true worst-case error, or any other kind of error could also be used exactly in the same way. The method, specially with the second algorithm, can easily be extended to the overdesign of phase characteristics, and can also be applied to other purposes than to only compensate for random errors in the component values. Deterministic effects, as the effects of losses and parasitic poles in active realizations can also be compensated.

\section{REFERENCES}

[1] H. J. Orchard, "Inductorless filters," Electronics Letters, vol. 2, June 1966, pp. 224-225.

[2] J. K. Fidler and C. Nightingale, "Slope-normalized sensitivity: an new sensitivity measure," Electronics Letters, vol. 15, January 1979, pp. 54-56.

[3] A. C. M. de Queiroz and L. P. Calôba, "Physically symmetrical and antimetrical ladder filters with finite transmission zeros," Proc. 30th MWSCAS, Syracuse, USA, 1987, pp. 639-643.

[4] A. C. M. de Queiroz and L. P. Calôba, "An approximation algorithm for irregular-ripple filters," Proc. STB/IEEE International Telecommunications Symposium, Rio de Janeiro, Brazil, 1990, pp. 430-433. 